\title{
Active Antenna System enhancement for supporting Licensed Shared Access (LSA) concept
}

\author{
Seppo Yrjölä, Eero Heikkinen \\ Nokia Solutions and Networks \\ Oulu, Finland \\ seppo.yrjola@nsn.com
}

\begin{abstract}
Mobile and wireless communication networks will need to cope with the tremendous increase in data traffic anticipated over the next decade. Beyond the levers of increased network densification and enhanced spectral efficiency more radio spectrum is clearly needed for mobile networks to fulfill capacity and coverage demands. Finding spectrum without existing incumbent usage in a harmonized manner becomes increasingly challenging. Spectrum sharing techniques can be used to optimize spectrum utilization across multiple services to share the same frequency band in a controlled manner. Another emerging technique to enhance cellular network capacity is smart antennas that can flexibly control radiation patterns. These new spectrum efficiency enhancements should be deployed in a way that requires minimum changes to the features and the architecture of the systems involved. In this paper, we concentrate on the Licensed Shared Access (LSA) concept which in recent years has received considerable interest in both European and USA regulation and standardization. We discuss how smart antennas technologies and Active Antenna System (AAS) in particular could be used to enhance LSA systems. Finally, we propose a system architecture and implementation scenarios for LSA that provides spectrum exclusion zone reduction and interference detection by using Active Antenna System.
\end{abstract}

Keywords- LSA, Licensed Shared Access, ASA, Authorised Shared Access, spectrum sharing, cognitive techniques, smart antennas, Active Antenna System, beamforming, sensing

\section{INTRODUCTION}

Mobile wireless communication networks will be facing the large increase in data traffic anticipated over the next decade. Beyond the levers of increased network densification and enhanced spectral efficiency more radio spectrum is clearly needed for mobile networks to fulfill capacity and coverage demands. In Europe, a total of around $600 \mathrm{MHz}$ of spectrum is currently allocated to mobile broadband, and significantly more additional spectrum will be needed towards 2020.The entire range of spectrum best suited for mobile applications between $400 \mathrm{MHz}$ and $6 \mathrm{GHz}$ is, however, already allocated to a number of different services and technologies, such as broadcast, aeronautical, satellite, defense, public safety and other commercial and non-commercial services; many of which do not utilize the spectrum intensively. There are several ways to get more spectrum for mobile networks. 'Clearing the spectrum', i.e., moving non-Mobile broadband (MBB) services away from their currently allocated spectrum bands is one straightforward way to free up more exclusive spectrum for MBB use. This has been best practice over the years, and will continue to be the preferred option for cellular mobile networks. In most cases, however, clearing spectrum requires significant investment and/or lengthy development time.

Shared use of spectrum has received a lot of attention as in some cases it may be, cost-wise and time-wise, a very efficient means to gain at least partial access to additional spectrum resources for mobile use. Mobile networks are based on predictable quality of service; therefore it is required that sufficient control mechanisms be implemented when applying spectrum sharing. In Licensed Shared Access (LSA) regulatory concept a license holders (incumbents) shares its spectrum with a limited amount of other service providers, LSA licensees under well-defined conditions in a way that interference is avoided [1]. There are bands in which utilization of spectrum is currently very low in time and/or location, thus offering attractive options for both the incumbent and an LSA user. The LSA approach is receiving growing attention as a potential means for speeding up the process of making International Mobile Telecommunications (IMT) bands available for Mobile Network Operators (MNO) in a timely manner to respond the market growth.

Another emerging technique to enhance cellular network capacity is smart antennas that can flexibly control radiation patterns [2] [3]. Smart antenna AAS is created by integrating several Radio Frequency (RF) components (power amplifiers and transceivers) - conventionally part of the base station - with the antenna's radiating elements. This enables the phase and amplitude of the signals from each radiating element inside the antenna to be electronically controlled, using signal processing to shape and steer the direction of the radiated beam vertically and horizontally. Benefits include more flexible deployment, improved capacity and coverage, smaller installation and lower power consumption, while also supporting a multi-technology network. Active antenna systems will improve existing base station site efficiency and performance. The technology can be used to create micro and macro cells simultaneously by using the same hardware located at the macro base station. Active antenna system technology is set to bring new levels of flexibility to the base station and create a customer experience matched to the needs of growing data traffic.

The focus of this paper is on LSA and AAS which both has rapidly gained a lot attention within the industry as well as regulation and standardization activities. Maximizing the availability of LSA spectrum to the LSA user and ensuring interference free operation to incumbent are vital for successful introduction of LSA. There is no prior work addressing 
specifically the AAS role related to these critical LSA success factors. Therefore in this paper, we introduce AAS for LSA usage scenarios and propose an architecture and implementation options for the LSA approach that takes into account the critical factors both from LSA licensee and incumbent perspectives. The rest of the paper is organized as follows. Section II introduces the LSA concept and the current status of LSA architecture. The AAS system is reviewed in Section III. In Section III, AAS usage scenarios are introduced. Based on these, the resulting enhanced architecture and implementations options are proposed in Section IV. Finally, conclusions are drawn in Section V.

\section{LICENSED SHARED ACCESS (LSA) CONCEPT}

Authorized Shared Access (ASA), originally proposed by an industry consortium, as being defined and reframed by European Commission (EC) Radio Spectrum Policy Group (RSPG) as Licensed Shared Access (LSA) is a regulatory approach to improve the efficiency of the spectrum usage by enabling two or more radio communication systems to share the same spectrum band in a fully controlled manner [4]. EC defines LSA as "A regulatory approach aiming to facilitate the introduction of radio communication systems operated by a limited number of licensees under an individual licensing regime in a frequency band already assigned or expected to be assigned to one or more incumbent users. Under the Licensed Shared Access (LSA) approach, the additional users are authorized to use the spectrum (or part of the spectrum) in accordance with sharing rules included in their rights of use of spectrum, thereby allowing all the authorized users, including incumbents, to provide a certain Quality of Service $(Q o S) "$ " [1].

Through this novel spectrum access model an incumbent primary license holder would grant spectrum access rights to one or more other users which may use the spectrum under specific rules and conditions. Rules and conditions defining how the spectrum may be used would be subject to individual agreements, and to permission from the National Regulatory Authority (NRA). The NRA would be expected to issue licenses to one, or a very limited number of mobile operators that would allow them to use specific bands as LSA licensees. Thereby binary usage in time or location should always be coordinated between the operators and the incumbent in order to assure performance predictability. Predictable levels of the quality of service (QoS) will strengthen mobile network operators' motivation for infrastructure investment compared to the scenario where usage is made available under a licenseexempt scheme such as a TV White Spaces.

LSA balances the needs of legacy spectrum users with those of operators, and it enables timely availability and licensed use of harmonized spectrum with predictable QoS. A major benefit envisioned with LSA is that the number of LSA licensees is limited, and that these LSA licensees are known to each other. Interference issues, if any, could be resolved and avoided either statically, through cooperative planning, or dynamically, through the use of common database access and cognitive radio technologies. Thus, in time, the concept may further evolve to embrace even more dynamic sharing principles.

An overview of the proposed LSA framework and concept is shown in Figure 1. Based on a commercial sharing agreement [5] the incumbent would share the data regarding the exact spectrum bands, the locations, and time when and where the frequency is available to the licensee in an LSA Repository. The LSA Controller manages the access to the spectrum based on policies and rules built upon LSA rights of use and information on the incumbent's use provided by the Repository. It retrieves information about available shared spectrum from the Repository through a secure and reliable communication path and propagates the permission or prohibition of use of the shared spectrum to the radio access network (RAN) and takes care for the right configuration of the corresponding parameters in the MNO's network, i.e. which base stations can make use of the LSA frequencies at what power levels and which base stations are not allowed to do so.

An important design factor in LSA architecture is the location of the LSA controller. Having the LSA Controller inside the domain of the MNO's network has several advantages in controlling and configuring the network parameters:

- Process requires deep insights into the MNO's radio access network.

- Configuration requires access to information that is business sensitive for the MNO.

- There are many parameters to be configured taking into account the entire network layout and interactions of Base Stations, which is best managed by the MNO also in order to avoid a real danger of "mis-configuration".

- To have control to optimize the traffic in MNO's network.

- There are various internal elements to a network that an externally managed Controller cannot and should not oversee.

However, the licensee should be responsible for compliance with technical requirements obtained from the incumbent such as meeting certain interference thresholds. This can best be accomplished via the Controller under the full control of the network operator. There could also be multiple LSA Controllers for each LSA licensee and the Controller can interface with one or multiple LSA Repositories.

In consideration of concrete LSA frequencies the 2300$2400 \mathrm{MHz}$ band is especially attractive because it is already an IMT band recommended by the ITU-R and is in use in some countries, e.g. in Asia, and is also specified as band 40 in 3GPP. It is widely supported in terminals including first attractive multi-mode multi band smart phones. In Europe this band is a good candidate for LSA as it is used in many countries limited in geography and/or time for e.g. governmental applications, including defense or wireless cameras. Through LSA it could be utilized for IMT mobile broadband at least locally or on a scheduled basis in countries where it is currently blocked entirely thus contributing to global spectrum harmonization. Standardization activities on 
the LSA concept are taking place in ETSI RRS [6] and initial discussions on potentially available spectrum with incumbents are already ongoing. In US spectrum sharing is also heavily discussed e.g. for 3550-3650 MHz band that is currently used by incumbent Federal Government radar systems and commercial fixed satellite systems. In 2012, the FCC made a first 3-Tier proposal for the prioritization of the users. First LSA implementations can be envisaged in the near future as first live network trials have already taken place with commercial base station products. World 1st on air LSA trial took place in Finland in April 2013 and reported in [7]. The trial was carried out with commercial 3GPP release 8 compliant radio access architecture, core network and network management system. Trial included full ecosystem, national spectrum regulator, incumbent and operator as well as the key control elements, LSA Controller and LSA Repository.

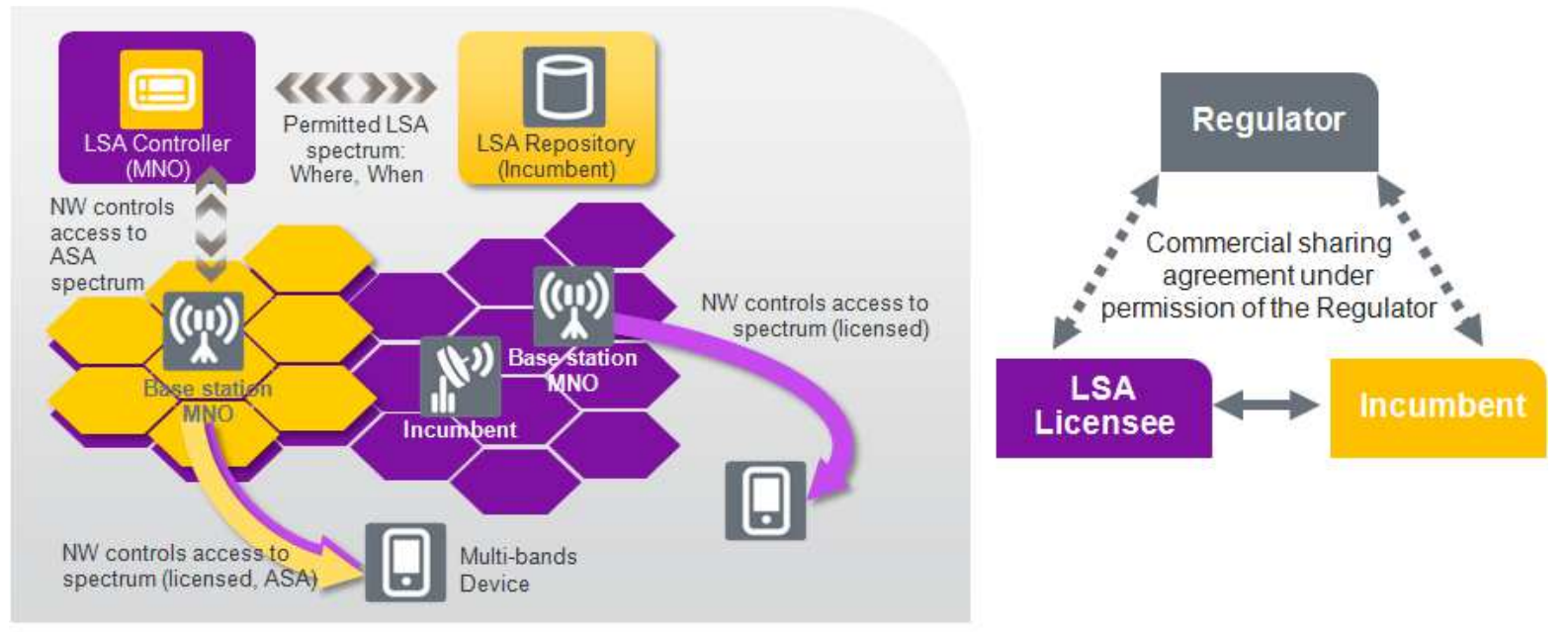

Figure 1. LSA framework and concept

\section{AAS CONCEPT}

The earliest base station antennas were basic omnidirectional assemblies radiating equally in all directions and providing relatively low capacity. As demand for mobile communications increased, sectorized antennas became more common. These created a specific radiation pattern, typically three-sector, and with sector-to-sector handoffs as devices moved within the macro-cell. Later still, antennas with integrated two passive cell-sectors inside one antenna radome became available, enabling six sector sites with three antennas for increased coverage and capacity. These antennas also featured mechanical tilt and, later, electrical tilt to adjust the radiation pattern to optimize handover and reduce interference. Remotely-controlled electrical tilt gives operators greater flexibility to improve network performance [3].

In the 3GPP LTE radio access network (RAN) AAS function as any other eNB. In addition to basic eNB base band processing Common Module (CM) split the Base Band IQ sample stream to multiple radios - as many streams as there are radios. After splitting the streams Common Module adjust phase and amplitude of each streams such that designed beam will be available from the antenna system as shown in Figure 2.

Precise control of the radiated pattern can only be achieved by the AAS in which one or a few radiating elements are individually controlled by an RF component. This provides much more control over coverage and capacity than the Remote Radio Heads and antenna systems in which all the radiating elements are fed by a single, common RF component. An active antenna system is typically of a similar size to a conventional passive antenna, yet offers much greater performance and brings major benefits to operators. These include more flexible deployment, improved capacity and coverage and lower power consumption, while also supporting a multi-technology network.

A principal advantage of active antennas is their ability to create and steer beams within the cell. Beamforming works by changing the phase and relative amplitude of the signal emitted from each radiating element, to create constructive or destructive interference. Constructive interference is used to amplify the beam in a given direction, while destructive interference is used to focus the beam, enabling it to be steered precisely. Beamforming can be applied to radiating and receiving antenna elements independently. Beamforming is a powerful technique, well-established for decades in military radar and other applications, that brings new flexibility to mobile networks. Operators can automatically adjust the sizes and positions of the cells to better serve the non-uniform demand from users across the macro-sector area. Highly flexible deployment is possible with beamforming, enabling an operator to cater for almost any demand profile within the macro-sector. 


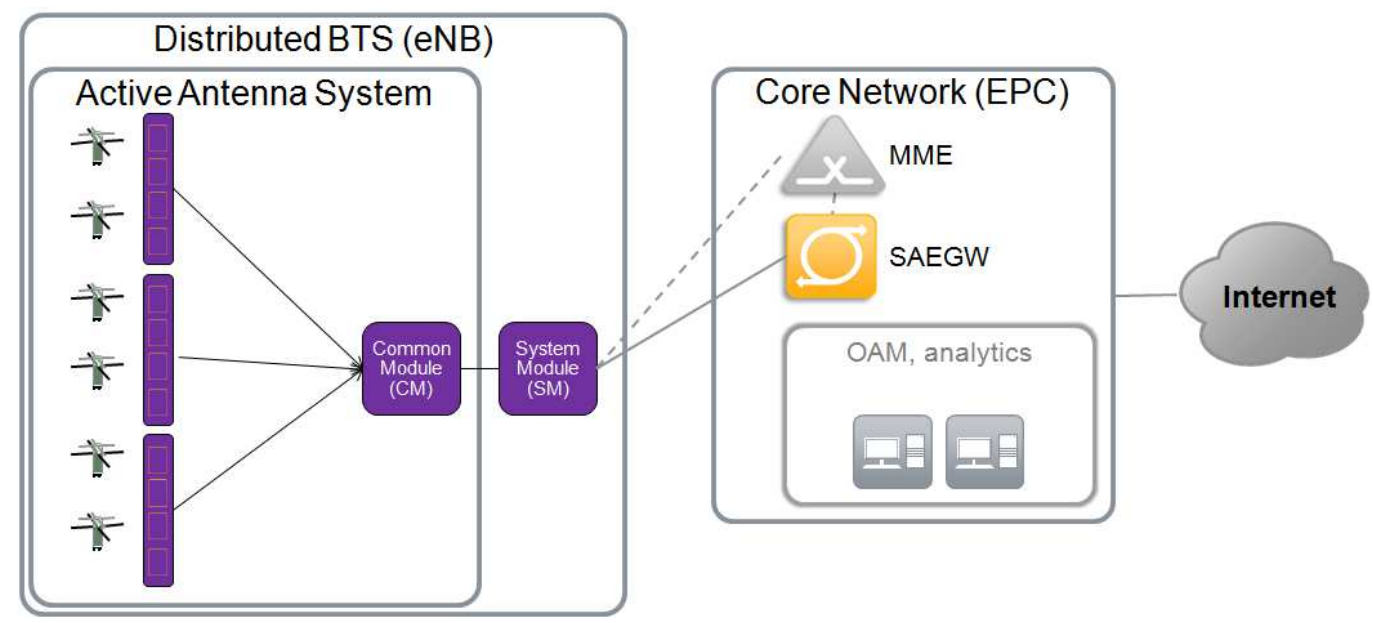

Figure 2. The basic AAS concept

AAS system enables several system level usage scenarios as shown in figure 3. [8][9].

- Unlike conventional non-active antennas that can only tilt all carriers, carrier-specific and separate RX/TX tilting can enable different carriers to be used to meet different demand scenarios.

- Similar to carrier-specific tilting, in system specific tilting active antennas can independently control the tilt of different technologies using the same frequency, for example GSM and WCDMA at $900 \mathrm{MHz}$ or GSM and LTE at $1800 \mathrm{MHz}$.

- In Multi-operator network sharing an active antenna system can be used to share transmission power between operators using the same antenna. Signals for different operators can be tilted and resources allocated independently to meet the needs of each operator according to subscriber density or other parameter.

- The high performance of active antennas can be further improved through the use of Self-Organizing Networks (SON) solutions. With the support of SON, dynamic steering of the beam becomes possible to distribute capacity precisely to where users need it when they need it. SON will enable the optimization of active antenna parameters according to actual traffic mix, traffic location and user demands. This will enable fully-automated user-tracking beamforming in which the beam follows the user and allocates resources to provide the best customer experience for each user at all times.
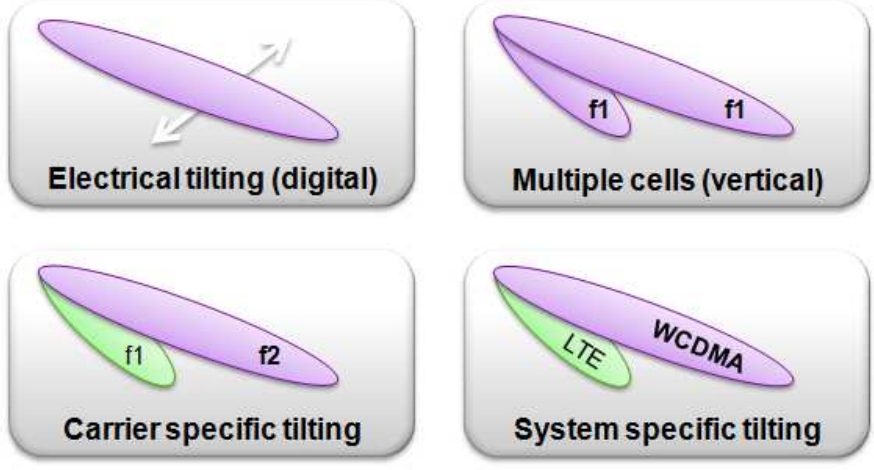
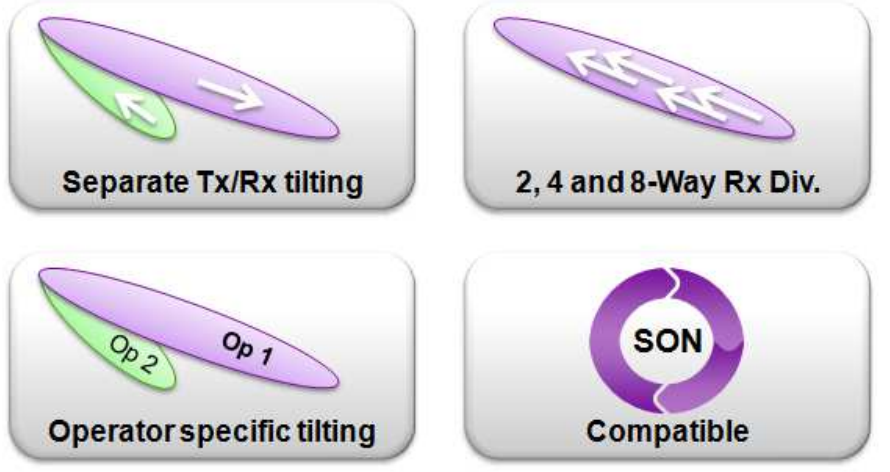

Figure 3. The basic AAS use cases 


\section{AAS USAGE SCENARIOS AND THEIR EFFECT ON LSA ARCHITECTURE}

\section{A. AAS for LSA usage scenarios}

The spectrum management of the currently mobile broadband networks is performed via static configurations based on mobile network planning data of the operator. The novel LSA radio access networks introduces need for semi static and dynamic configurations of the spectrum resource because LSA spectrum needs to be both taken into use and in particular evacuated according to predefined terms and conditions if requested by the incumbent user. Current static mobile broadband spectrum allocation methods using traditional exclusive spectrum need to be complemented with a new method where occasionally in some regions and time certain parts of the spectrum may no longer be exclusive.

Key characteristic of LSA is that according to agreement between incumbent and LSA licensee the incumbent may request specific LSA resources for own usage. Request could be defined by static rules e.g. a defined exclusion zone and/or time where the spectrum is used by the incumbent or dynamic rules e.g. evacuation of spectrum currently used by MNO on request from the incumbent for emergency situations. In both cases zones where spectrum use under LSA is not allowed are defined by geographical area, time and transmitter/receiver characteristics.

The Operator has to take appropriate measures in the network e.g. switch off or reconfigure the LSA spectrum of specific Base Stations and cells without violating the evacuation lead time in case of spectrum resource evacuation actions triggered by the incumbent. In general such measures are easy to realize. LSA repository defines or will define dynamically a reservation zone which needs to be evacuated on request.

Key criteria for the LSA architecture is how to allocate and de-allocate the LSA spectrum in way that minimize the size of exclusion zones and maximize spectrum made available for Mobile Operators while avoiding interference to incumbent system and maintaining mobile end user experience to extent possible.

\section{1) AAS for LSA exclusion zone reduction}

Active antenna system technologies can reduce potentially very large LSA exclusion zone and potential interference as shown in the Figure 4. Instead of shutting down the whole LSA base station or cell, AAS can modify radiation pattern per cell. Modified radiating pattern is enough to prevent interference to the incumbent.

Using AAS mobile operators can automatically adjust the sizes and positions of the cells to better utilize the non-static LSA spectrum to serve non-uniform demand from users across the macro-sector area.

Carrier-specific tilting can enable different carriers to be used according to LSA spectrum availability. For example tilting the carrier used for LSA enables same frequency to be used simultaneously for the incumbent service. System specific tilting of active antennas can independently control the tilt of different technologies enabling the continuous usage of radio equipment in the case of LSA band becomes not available.

In multi-operator network sharing an active antenna system can be used to share transmission power between operators using the same antenna. In LSA case novel use case could be sharing the radio site by incumbent and LSA licensee as signals for different operators can be tilted and resources allocated independently to meet the needs of each operator according to agreement. For example the incumbent can use TD-LTE network and radio equipment for their program making (wireless cameras) while the LSA licensee can utilize free zones for their normal TD LTE mobile broadband traffic.

In LSA case with the support of SON, dynamic steering of the beam becomes possible to distribute capacity precisely to where users need, when they need it and where ASA spectrum is available.
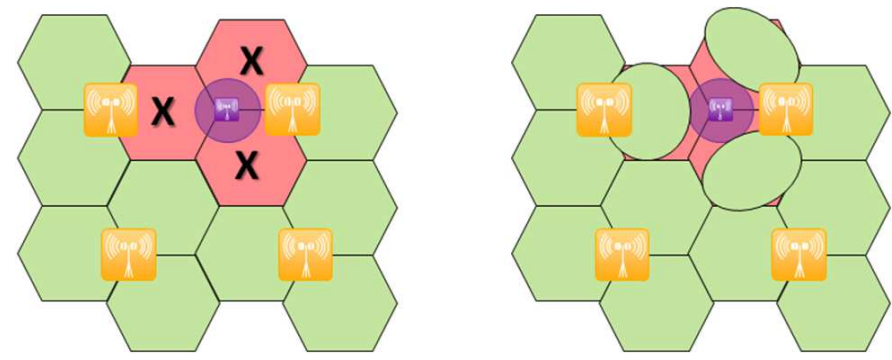

Figure 4. AAS for LSA exclusion zone reduction

\section{2) AAS for RF monitoring and interference detection}

The discussed LSA solutions can be further improved through utilizing Active Antenna System for RF monitoring and interference detection of the LSA band by configuring the TX feedback loop of the AAS. LSA sensing could be used to improve the accuracy of the LSA repository by providing measurement data of the LSA spectrum utilization in the BTS area as shown in the Figure 5. The measured exact spectrum usage data related to incumbent could be used for restriction area/guard band calculation in addition to static exclusion zone tables or equation in the ASA repository and controller.

In addition AAS interference detection could be used to add extra security to guarantee interference free operation for the incumbent by continuous spectrum measurements and analysis. Radios collect spectrum using in band or out of normal operating band data.

Basic function of the Common Module (CM), in Figure 6. is to implement digital beam forming. Input for the $\mathrm{CM}$ is coming from the radios and from the System Module (SM). For uplink direction CM combines phase and amplitude adjusted signals from each radio per carrier. For downlink direction CM splits and adjusts amplitude and phase of each radio and carrier. 
$\mathrm{CM}$ is capable of receiving both in-band and out-of-band signals. By phasing those signals CM can exactly detect if there is LSA activity in given direction. If there are independent RX radios then common module can measure, synchronize and phase data such, that more accurate direction of the interferer can be detected. CM sends analyzed spectrum info to ASA controller for decision making.

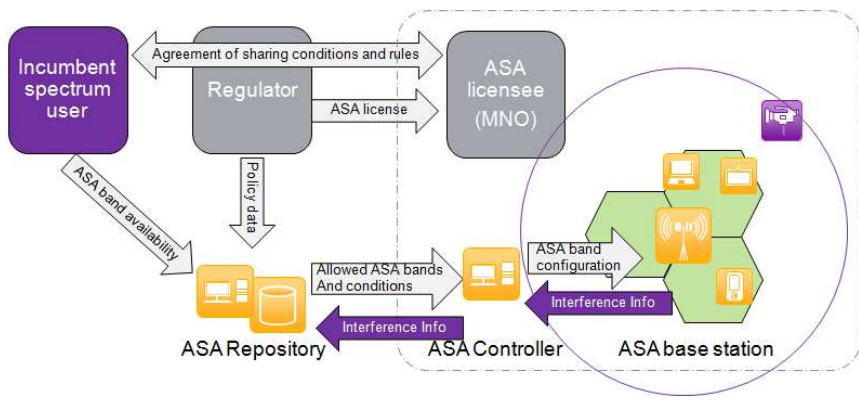

Figure 5. AAS for RF monitoring and interference detection

\section{B. AAS implementation scenarios in the LSA architecture}

\section{1) AAS beamsteering for LSA exclusion zone reduction}

In the AAS each radio in the system is calibrated in such a way that exact phase, delay and amplitude of the radio are known. Using this knowledge and knowing AAS antenna geometry, it is possible to create independent beams which can be directed to the needed direction within AAS physical antenna field of view. Creation of the beams is done in one common beam-forming function in so called common module (CM) as shown the Figure 6. Physically beam-forming is done by phasing, delaying and adjusting amplitude of each radio such that combined signal is directing to desired angle.

The performance of active antennas is controlled by OAM system and can be further improved through the use of SelfOrganizing Networks (SON) solutions. With the support of SON, dynamic steering of the beam becomes possible to distribute available capacity precisely to where users need it when they need it while minimizing LSA restriction zones needed to protect incumbent user. SON will enable the optimization of active antenna parameters according to the available spectrum resource, actual traffic mix, traffic location and user demands.

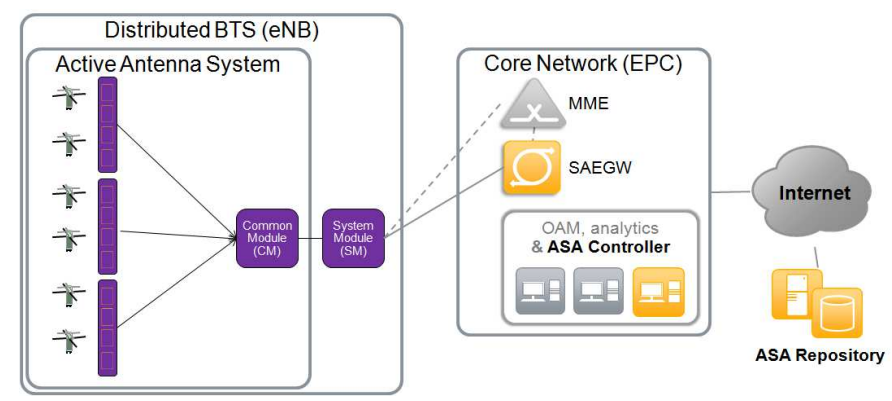

Figure 6. AAS beam steering architecture

\section{2) LSA workflow with $A A S$}

The LSA basic workflow with AAS enhancements will be the following:

Preparation

1. Assess the balance between demand/current network load and existing spectrum resources

2. Estimate the business potential from sharing of spectrum from others.

ASA licensing

3. Define and agree rules and conditions with regulator and incumbent

4. Licensing agreement between regulator and $\mathrm{MNO}$

ASA deployment

5. MNO obtains knowledge of available parts of the LSA band and usage conditions in the given area from a LSA repository database

6. MNO does network planning for the LSA band

7. MNO deploys a network that supports the LSA band

- OAM commands to AAS system module for beam steering

8. MNO reports the LSA repository which parts of the ASA band it starts to use

9. ASA controller and OAM configures the LTE base stations to specific parts of the LSA band

- OAM command to AAS system module for beam steering

10. MNO moves user to the parts of the LSA band from its other networks

11. QoS measurements

- With optional AAS RF monitoring and interference measurement

12. Balance capacity demand and supply between different networks

13. Customer experience management (CEM) using the LSA band

ASA release

14. Stop transmissions on parts of the LSA band if regulator or incumbent requests it or durations of licensing agreement expires

- Use AAS to reconfigure antenna beams to evacuate LSA band where requested by incumbent.

Pre-conditions:

- LSA band is deployed, user initiates the release

- To be used OAM like feature Graceful shutdown is pre-enabled per each BTS enhanced with LSA band

- Sites DB maintains status of list of BTS/cells enhanced with LSA. 


\section{3) AAS for LSA sensing architecture}

Active antenna can be used to monitor out of band traffic or active antenna can monitor direction of interferers within operating band. This information can be used in LSA radio system to alarm users arriving to the band. AAS can also assist frequency selection process in LSA controller by detecting spectrum usage of out of AAS operating band.

If the expected LSA interferer is in AAS RX band, then interference can be collected using RX radios and accurate direction of interferer can be calculated in common module.

If the expected interferer is in AAS TX band or if interferer is out of band, then AAS radios will be configured to receive at interferer transmitter band. Or AAS can receive at wide band including the expected interferer transmission.

By measuring time (phase) difference between detected signals from any user, it is possible to accurately detect user location as shown in the Figure 7. User can be an incumbent or a mobile. Each AAS radio detect interferer radio signal independently and CM calculates interferer location using detected phase differences.

Sampling of the interferer does not need to be continuous.

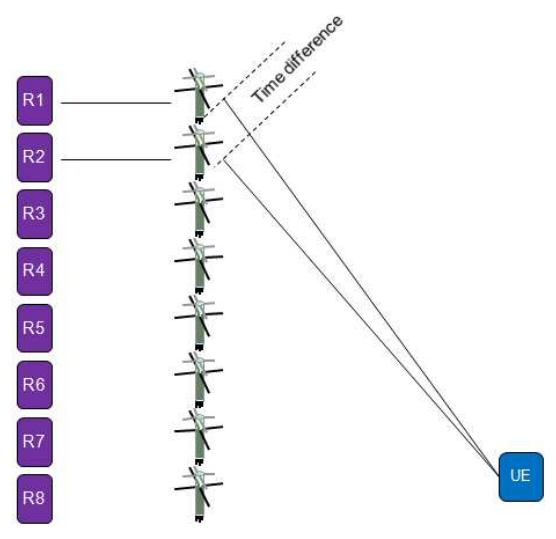

Figure 7. AAS for LSA sensing architecture.

ASA controller will through OAM select a band which will be monitored by AAS common module. AAS common module then sends an alarm if there is interference detected in the given band.

If AAS use ASA band for cellular operation, then CM shall be configured to detect non expected interference rise in given band and CM shall alarm through the ASA Controller and ASA repository that there might be a primary user in that band. CM shall also scan direction of interferer and see if there is interference rise at own system idle period.

Detailed measurement flow will be the following:

- Configure TX feedback to the ASA band. Configuration is controlled by $\mathrm{CM}$

- $\quad$ By-pass RF filter using switch (SW)

- Use TX DPD feedback radio to collect data inside and outside of operating band
- Send collected data to the common module with timing information

- If expected signal is at low level then CM shall stop own TX for the measurement period

- Accuracy of this measurement is the antenna radiation pattern. Alternatively to TX feedback radio, separate $\mathrm{RX}$ radios may be used

- If there is a separate RX radio used, instead of TX DPD feedback radio, then concurrent measurements could be used

- If concurrent measurements are done, then more accurate direction of interferer can be detected.

\section{CONCLUSIONS}

Mobile and wireless communication networks will need to cope with the tremendous increase in data traffic over the next decade. Beyond the levers of increased network densification and enhanced spectral efficiency, more radio spectrum is clearly needed for mobile networks to fulfill capacity and coverage demands. As finding spectrum without existing incumbent usage in a harmonized manner becomes increasingly challenging, effort is being placed on finding methods to share the same frequency band in a controlled manner.

In this paper, we focused on LSA and AAS which both has rapidly gained a lot attention within the industry as well as regulation and standardization activities. Maximizing the availability of LSA spectrum to the LSA user and ensuring interference free operation to incumbent are vital for successful introduction of LSA. We introduced an enhanced system architecture for LSA that provides spectrum exclusion zone reduction and interference detection by using Active Antenna System. We then proposed an enhanced AAS beam steering architecture for LSA that reduced needed guard/evacuation zone area in spectrum allocation and evacuation while enables easy integration in current networks based on standardized interfaces. Finally we introduced AAS sensing architecture with implementation scenarios that provides extra protection toward interference, improves accuracy and reduces the area of the calculated restriction zones while utilizes existing AAS radio HW implementations.

This study showed promising LSA architecture enhancements with AAS. Future work will concentrate on the air field trialing proposed concepts and implementation scenarios in the CORE+ LSA AAS trial network.

\section{ACKNOWLEDGMENT}

The authors would like to acknowledge CORE+ project research consortium that consists of VTT Technical Research Centre of Finland, University of Oulu, Centria University of Applied Sciences, Nokia Solutions and Networks, PehuTec, EXFO, Elektrobit, Anite, Rugged Tooling, Finnish Defence Forces, Finnish Communications Regulatory Authority, and Tekes - the Finnish Funding Agency for Innovation. 


\section{REFERENCES}

[1] RSPG, "RSPG Opinion on Licensed Shared Access", RSPG13-538, European Commission, Radio Spectrum Policy Group, Nov. 2013."

[2] O. Yilmaz, S. Hämäläinen and J. Hämäläinen, "Analysis of Antenna Parameter Optimization Space for 3GPP LTE", Vehicular Technology Conference Fall (VTC 2009-Fall), 2009 IEEE 70th.

[3] G.V. Tsoulos, "Smart antennas for mobile communication systems: benefits and challenges" Electronics \& Communication Engineering Journal , pp. 84-93, Apr. 1999.

[4] RSPG, "Report on Collective Use of Spectrum (CUS) and other spectrum sharing approaches", RSPG11-392, European Commission, Radio Spectrum Policy Group, Nov. 2011.

[5] CEPT, "Licensed shared access", Draft ECC Report 205, Oct. 2013.

[6] ETSI RRS, "Mobile Broadband services in the 2300-2400 $\mathrm{MHz}$ frequency band under Licensed Shared Access regime," TR $103.113 \mathrm{v}$ 1.1.1, July 2013.
[7] M. Matinmikko, M. Palola, H. Saarnisaari, M. Heikkila, J. Prokkola, T. Kippola, T. Hänninen, M. Jokinen, and S. Yrjölä, "Cognitive Radio Trial Environment: First Live Authorized Shared Access-Based SpectrumSharing Demonstration, " IEEE Vehicular Technology Magazine, vol. 8, no. 3, , pp. 30-37, Sept. 2013.

[8] O. Yilmaz, S. Hämäläinen and J. Hämäläinen, ”System level analysis of vertical sectorization for 3GPP LTE", in IEEE International Symposium on Wireless Communication Systems (ISWCS), pp. 453-457, Oct. 2009.

[9] D. Laselva, Z. Altman, I. Balan, A. Bergström, R. Djapic, H. Hoffmann, L. Jorguseski, I. Z. Kovács, P. H. Michaelsen, D. Naudts, P. Ramachandra, C. Sartori, B. Sas, K. Spaey, K. Trichias, Y. Wang, "INFSO-ICT-316384 SEMAFOUR D4.1, SON functions for multi-layer LTE and multi-RAT networks (first results)", 2013. 